\title{
COMPUTATION OF WORST-CASE PILOT INPUTS FOR CLEARANCE OF FLIGHT CONTROL LAWS
}

\author{
Prathyush P. Menon* Declan G. Bates* \\ Ian Postlethwaite* \\ * Control and Instrumentation Research Group, \\ Department of Engineering, \\ University of Leicester, Leicester, U.K., LE1 7RH \\ Email: ppm6,dgb3,ixp@le.ac.uk
}

\begin{abstract}
In this paper, global optimisation and nonlinear simulation are used to search for the worst-case control demands which may be input by a pilot flying a modern high performance aircraft with a full authority flight control law. The flexibility of global optimisation is exploited to simultaneously search for worst-case pilot inputs and worst-case combinations of uncertain parameters in the nonlinear aircraft model. The importance of explicitly considering uncertainty in the aircraft model is clearly demonstrated by the results obtained. Copyright IFAC 2005
\end{abstract}

Keywords: Flight control, Global Optimization, Robustness Analysis, Simulation

\section{INTRODUCTION}

Modern high performance aircraft are often designed to be naturally unstable (or to have reduced natural stability margins) for performance reasons, such as to improve manoeuvrability or to decrease drag and fuel consumption. Such aircraft can therefore only be flown by means of a flight control law, which provides artificial stability, and is hence a safety-critical system. The main difficulties faced by flight control law designers are associated with nonlinearity and uncertainty in the aircraft dynamics. At high angles of attack (AoA) or at high rotation rates, aircraft flight dynamics become highly nonlinear, due to significant levels of cross-coupling between axes. Also, all aircraft have significant nonlinearities, associated with limitations in the movement of aerodynamic control surfaces, which can sometimes be excited by large pilot input demands. Significant levels of uncertainty are also inevitably present in even the most detailed aircraft simulation model, so that a large number of "uncertain" parameters will be used to model variations in configuration, aerodynamic, sensor and actuator parameters.

The search for "worst-case" pilot control inputs is an important part of the certification (also called clearance) process for any new flight control system. The definition of "worst-case", of course, depends on the particular clearance criteria that is being considered. For highly agile combat aircraft, a key consideration is the identification of socalled departure susceptibility - the computation of pilot inputs that will excite the nonlinear aircraft dynamics to such an extent as to lead to loss of stability and/or controllability. For flight control laws equipped with a Manoeuvre Load Limiter (MLL), on the other hand, pilot inputs that test the robust functionality of the envelope protection system are required to be computed. A number of recent studies have considered the above problem. In (RyanIII, 1995), the departure susceptibility of the X-31 Enhanced Fighter Manoeuvrability demonstrator aircraft was evaluated. Genetic Algorithms (GA's) were used to 
Table 1. Aircraft Model Uncertain Parameters

\begin{tabular}{lll}
\hline Parameter & Bound & Description \\
\hline$\Delta_{\text {mass }}$ & {$[-0.1+0.1]$} & variation in aircraft mass from nominal one $(9100 \mathrm{~kg})[\%]$ \\
$\Delta_{x c g}$ & {$[-0.075+0.075]$} & variation in position of center of mass [m] \\
$\Delta_{C_{m_{\delta_{e}}}}$ & {$[-0.05+0.05]$} & uncertainty in pitching moment due to elevator deflection $[1 / \mathrm{rad}]$ \\
$\Delta_{I_{y y}}$ & {$[-0.2+0.2]$} & uncertainty in aircraft inertia around y-axis from nominal one $\left(81000 \mathrm{~kg} \cdot \mathrm{m}^{2}\right)[\%]$ \\
$\Delta_{C_{m_{\alpha}}}$ & {$[-0.05+0.05]$} & uncertainty in pitching moment due to AoA [1/rad] \\
\hline
\end{tabular}

search for pilot inputs that maximised a cost function associated with aircraft departures - the absolute sum of certain states of the system, such as attitude rates, AoA and sideslip angle. This was further developed in (Menon et al., 2003) using a multi-modal genetic search with an energy-like cost function, and applied to the nonlinear simulation model of the Indian Light Combat Aircraft, (Chetty et al., 2002). Neither of the above studies, however, considered any form of uncertainty in the aircraft simulation model. A different, but related, approach to the same problem is reported in (Forssell and Hyden, 2003). In these studies, a particular sequence of pilot inputs called the Clonk manoeuvre, developed by SAAB using piloted simulation testing to detect the proneness for departure of the Gripen aircraft, was applied to the ADMIRE, (Forssell et al., 2001), simulation model. Global optimisation methods were used to compute the worst-case combination of uncertain aircraft parameters.

In this paper, a global optimisation method is used to simultaneously search for the worst-case pilot inputs and the worst-case uncertain parameter combination for the ADMIRE model with an industry standard full authority flight control law. Following (Forssell and Hyden, 2003), the cost function used in this study is the maximum value of AoA over a finite time period. The main contributions of the paper are to show that (a) global optimisation methods easily allow worst-case pilot inputs and uncertain parameter combinations to be computed simultaneously, (b) optimistic (i.e. unreliable) results will be generated if they are computed separately, and (c) global optimisation methods easily find very simple combinations of pilot input signals which have worse effects than the aggressive Clonk manoeuvre, which was developed via extensive piloted simulation trials.

\section{ADMIRE AIRCRAFT MODEL}

The aircraft model considered in the present study is ADMIRE (Aero-Data Model In a Research Environment) a non-linear, 6 degree of freedom simulation model (Forssell et al., 2001), developed by the Swedish Aeronautical Research Institute (FOI) using aero data obtained from a generic single seated, single engine fighter aircraft with a delta-canard configuration. ADMIRE is augmented with a full authority industrial standard flight control system. The model includes a large number of uncertain aerodynamic, actuator, sensor and inertia parameters, whose values, within specified ranges, can be set by the user.

The aircraft dynamics are modelled as a set of twelve $1^{\text {st }}$ order coupled nonlinear differential equations and given as

$$
\dot{x}(t)=f(x(t), u(t), \Delta) ; y(t)=h(x(t), u(t))
$$

where $x(t)$ is the state vector with 12 components, i.e., velocity, AoA, sideslip angle, angular rate, attitude, and position vectors. $\Delta$ represents the uncertain parameters in the aircraft simulation model. $y(t)$ is the output vector, and $u(t)$ is the control input vector(Forssell et al., 2001). The control input is determined by:

$$
u(t)=g\left(x(t), y_{\mathrm{REF}}(t)\right)
$$

where $g(\cdot, \cdot)$ is the flight control law, and $y_{\mathrm{REF}}(t)$ is the reference demand consisting of the pilot inputs. The present study considers pitch and roll stick inputs only, and the amplitude of inputs is limited to \pm 40 Newtons. Table 1 gives details of the uncertain parameters considered in this study. Equations (1) and (2) together represent the closed loop dynamics of the aircraft.

The augmented ADMIRE operational flight envelope is defined up to Mach 1.2 and altitude 6000 meters. The longitudinal control law is gain scheduled over the whole flight envelope with respect to Mach and altitude variations and is designed to ensure robust stability and handling performance. The model also contains actuator rate limiting and saturation blocks as well as nonlinear stick shaping elements.

\section{GLOBAL OPTIMIZATION}

The global optimisation approach applied in this paper is based on GA's, which are general purpose stochastic search and optimisation procedures that use genetic and evolutionary principles (Goldberg, 1989). In a genetic search technique, a randomly sourced population of candidates undergoes a repetitive evolutionary process of reproduction through selection for mating according to a fitness function, and recombination via crossover 
Table 2. Pilot control input discretization levels and binary representation

\begin{tabular}{lll}
\hline Pitch Stick & Roll Stick & Binary \\
\hline Full MAX [+40N] & Full MAX [+40N] & 11 \\
Half MAX [+20N] & Half MAX [+20N] & 10 \\
Half MIN [-20N] & Half MIN [-20N] & 01 \\
Full MIN [-40N] & Full MIN [-40N] & 00 \\
\hline
\end{tabular}

with mutation. A complete repetitive sequence of these genetic operations is called a generation. The candidates are encoded as artificial chromosomes, and a fitness function is defined to assign a performance index to each candidate - this function is specific to the problem and is formed from the knowledge domain.

Each optimization variable, the gene, is binary coded according to an accuracy level and combined sequentially to form the chromosome, which represents a potential candidate solution. The search starts from an initial random number of candidates of fixed size $N_{\text {size }}$, presently fixed at 50. The candidates from the current generation are qualified to produce the successive generations depending on a selection scheme. A roulette wheel selection scheme with a selection probability of 0.6 is applied in this study. During crossover, the recombination operator ensures mixing up of the information content between two different binary coded chromosomes. A single point crossover with a probability of crossover 0.9 is used here. The point of crossover is determined randomly over the length of bits. Mutation introduces random variations in the population in the search space, by randomly flipping a bit value. The probability of mutation is kept low and fixed at 0.05. The number of maximum generations is the termination criterion and is fixed at 100 generations.

GA's has become a popular, robust search and optimisation technique for problems with large as well as small parameter search spaces. Due to their stochastic nature, GA's can be expected to have a much better chance of converging to a global optimum. A recent survey (Fleming and Purshouse, 2002) provides examples of the many applications of GA's in the control engineering field. The reader is referred to (Goldberg, 1989) for more details of GA operators, binary coding schemes and the theory of genetic search.

\section{PROBLEM SETUP AND ANALYSIS}

Five different sets of analysis results are presented in this paper:

Analysis I: Nominal Clonk analysis. For the nominal model, the pilot control inputs $y_{R E F}(t)$ specified by the Clonk manoeuvre over the time period $\tau, \tau \in\left[\tau_{0} \tau_{f}\right]$, are input and the maximum value of the the chosen cost function is computed.

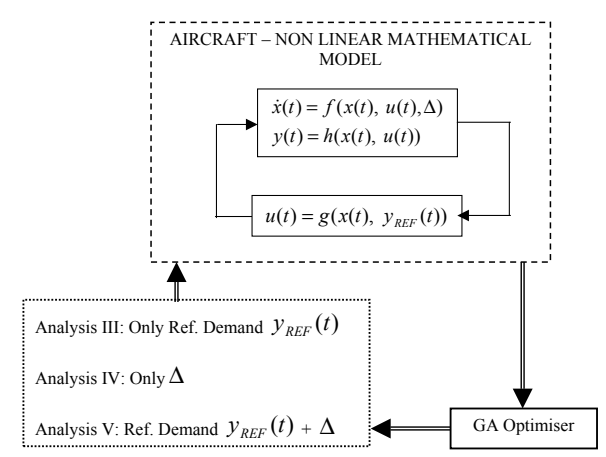

Fig. 1. The framework for analysis

Analysis II: Uncertain Clonk analysis. For the uncertain simulation model, for the pilot control inputs $y_{R E F}(t)$ specified by the Clonk manoeuvre over the time period $\tau, \tau \in\left[\tau_{0} \tau_{f}\right]$, the combination of uncertain parameters, $\Delta$, that maximises the chosen cost function is computed.

Analysis III: Worst-case pilot inputs for nominal model. For the nominal model, the pilot control inputs $y_{R E F}(t)$ over the time period $\tau, \tau \in\left[\tau_{0} \tau_{f}\right]$, that maximise the chosen cost function are computed.

Analysis IV: Worst-case uncertain parameters for fixed pilot inputs. For the uncertain simulation model, for the worst-case pilot control inputs $y_{R E F}(t)$ computed in Analysis III, the combination of uncertain parameters, $\Delta$, that maximises the chosen cost function is computed.

Analysis V: Worst-case pilot inputs and uncertain parameters. For the uncertain simulation model, the combination parameter uncertainties, $\Delta$, and the pilot control inputs $y_{R E F}(t)$, over the time period $\tau, \tau \in\left[\tau_{0} \tau_{f}\right]$, that maximizes the chosen cost function is computed simultaneously.

Analyses I and II are similar to those performed in (Forssell and Hyden, 2003), and are given here mainly for comparison. For other analysis tasks, a common framework consisting of the global optimizer and the nonlinear model of ADMIRE was used. Figure 1 shows a block diagram representing the problem set-up. Depending on the type of analysis required, the GA optimizer provides the appropriate input to the nonlinear simulation model.

In order to reduce the level of complexity, the continuous search space is discretized into a number of possible amplitude levels as shown in table 2 . This is consistent with current industrial practice, whereby step and doublet inputs of predefined amplitude levels are often used to clear the flight control laws, (Menon et al., 2003). The time axis is also discretized, in the sense that changes in the magnitude of pilot inputs are allowed to occur only at regular intervals of one second, and 

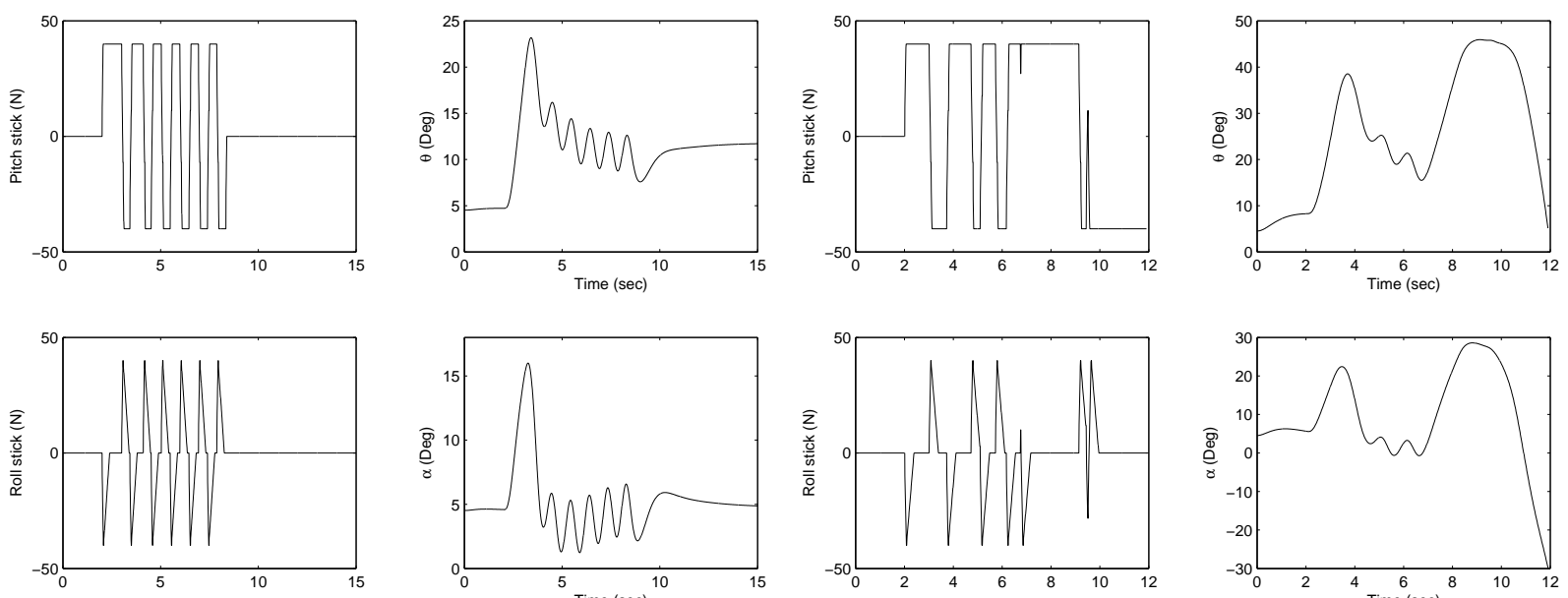

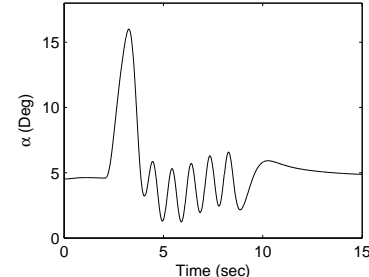

(a)
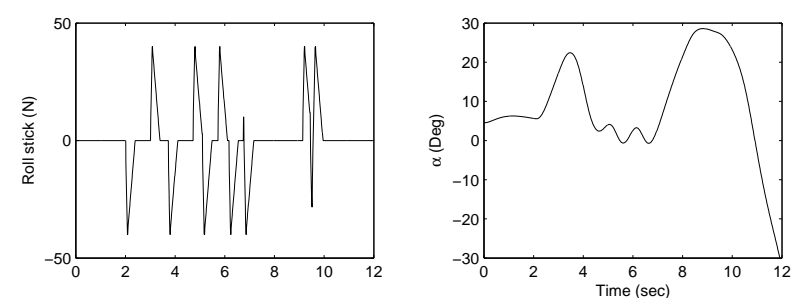

(b)

Fig. 2. Clonk analysis results about $M=0.4$ Altitude $=3000$, Level Trim; a) Analysis I b) Analysis II

values of pilot inputs are held constant for at least one second. A 1 sec input frequency is high enough to test the controls but still low enough to remain a realistic input frequency for a pilot (RyanIII, 1995). Two bits can represent each pilot input signal over each one second interval of time, for the discretization as given in table 2 .

For analysis III, each chromosome is of length 20 bits - an arbitrary manoeuvre of 5 seconds duration. At the end of the $5^{t h}$ second, all the control inputs are brought to zero and the simulation is continued for another 5 seconds. Each simulation is ofof 10 seconds. The maximum AoA over the 10 second time history is the fitness associated with each chromosome. In Analysis IV only the uncertain parameters $(\Delta)$ are considered and $y_{R E F}(t)$ is fixed to one particular manoeuvre. The $\Delta$ define a hyperbox of dimension 5 , and each uncertain parameter defines a gene for the problem considered. The chromosome length depends on the accuracy, fixed at 1e-06 presently. With this accuracy, each chromosome is of length 105 bits, consisting of 5 genes each of 21 bits. In Analysis V, in order to simultaneously search for the worst-case pilot inputs and the worst-case uncertain parameter combination, the chromosome is of length 125 bits, in which 20 bits consist of the pilot control input as in Analysis III, and the remaining 105 bits consist of $\Delta$ as in Analysis IV.

\section{RESULTS}

All results shown are about a flight condition of 0.4 mach, $3000 \mathrm{~m}$ height and level trim.

\subsection{Analysis I}

For the purposes of comparison with previous studies we first of all present results using the
Clonk manoeuvre. According to the specifications for the Clonk manoeuvre, (Forssell and Hyden, 2003), the pilot's pitch stick command switches, with a limited rate, between its maximum magnitude limits when the pitch attitude reaches its maximum or minimum. The roll stick command is simultaneously switched to the opposite extremum to that of the pitch stick command. However, once the roll stick reaches an extremum, it immediately starts moving in the opposite direction at a defined rate, called the roll return rate. The pitch stick command, on the other hand, remains for some additional time on its magnitude limit - this time period is referred to as the pitch stick delay. At the next occurrence of a maximum or minimum of the pitch attitude the next switching for both the stick commands occurs, and this sequence is then repeated for a specified period of time.

Figure 2(a) shows the pilot input commands generated by the Clonk manoeuvre. For the Clonk analysis, a pitch and roll stick deflection rate of $720 \mathrm{~N} / \mathrm{sec}$ and $500 \mathrm{~N} / \mathrm{sec}$, respectively, and a pitch stick delay and roll return rate of 1 sec and 128 $\mathrm{N} / \mathrm{sec}$, respectively, were recommended by the developers of the ADMIRE model, (Forssell, 2004), and were used in this study. Figure 2(a) shows the corresponding AoA and pitch angle time history - the maximum AoA achieved was $16.0038^{\circ}$. Neither actuator magnitude saturation nor any considerable actuator rate limiting were observed for this sequence of pilot inputs.

\subsection{Analysis II}

In this analysis, pilot control inputs determined by the Clonk manoeuvre were applied, while the GAbased optimisation software was used to compute the worst-case combination of uncertain parame- 


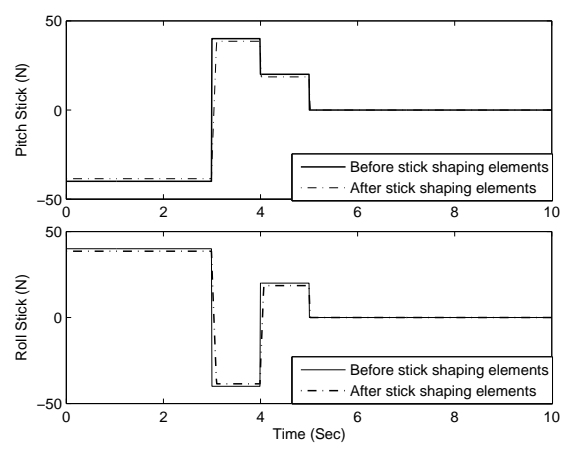

(a)

Fig. 3. Analysis III and Analysis IV results

Table 3. Analysis II results

\begin{tabular}{llllll}
\hline$\left[\begin{array}{ccccc}\Delta_{\text {mass }}^{*} & \Delta_{x c g}^{*} & \Delta_{C_{m_{\delta_{e}}}}^{*} & \Delta_{I_{y y}}^{*} & \Delta_{C_{m_{\alpha}}}^{*}\end{array}\right]$ & $\max \alpha(t)$ \\
\hline$\left[\begin{array}{lllll}0.0413 & 0.0750 & 0.0499 & -0.1218 & 0.0500\end{array}\right]$ & 28.6008 \\
\hline
\end{tabular}

ters. The results of this analysis are shown in Figure 2 (b) and Table 3. As expected, the effect of considering uncertainty in the aircraft simulation model has been to significantly increase the maximum value of AoA achieved.

\subsection{Analysis III}

In analysis III, worst-case pilot control inputs are computed for the nominal model. The maximum AoA obtained from the analysis is $50.0233^{\circ}$. Figure $3(\mathrm{a})$ shows the corresponding pilot control inputs while Figure 3(b) shows the AoA time history, given in continuous bold lines. Note that the maximum AoA is significantly higher than the one obtained by using the pilot inputs determined by Clonk manoeuvre in both Analyses I and II.

\subsection{Analysis IV}

In this analysis, the worst-case pilot control inputs generated by analysis III are used, while the GA-based optimization searches for the worstcase combination of uncertain parameters $\Delta$. This corresponds to searching for the worst-case pilot inputs and uncertain parameters separately. The uncertain parameter combination given in Table 4 gave the maximum AoA $\left(61.7187^{\circ}\right)$ for this approach. The dotted graph shown in Figure 3(b) shows the AoA time history. Note that the worstcase values of two of the uncertain parameters are inside the hyperbox that defines the search space. Interestingly, if the value of $\Delta_{x c g}$ is changed to be at its maximum allowable value (which would correspond to the "worst-case" value most likely suggested by an intuitive interpretation of flight mechanics principles), the maximum AoA build

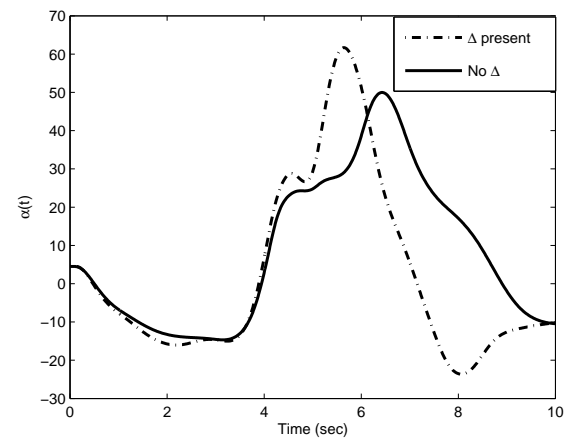

(b)

Table 4. Analysis IV results

\begin{tabular}{|c|c|c|c|c|c|}
\hline$\Delta_{\text {mass }}^{*}$ & $\Delta_{x c g}^{*}$ & $\Delta_{C_{m_{\delta_{e}}}}^{*}$ & $\Delta_{I_{y y}}^{*}$ & $\Delta_{C_{m_{\alpha}}}^{*}$ & $\max \alpha(t)$ \\
\hline$[0.1000$ & 0.0104 & -0.0500 & -0.2000 & $0.0429]$ & 61.7187 \\
\hline
\end{tabular}

up turns out to be only $48.7636^{\circ}$. This result shows the limitations of relying entirely on flight mechanics intuition when analysing highly nonlinear flight control problems. It also illustrates the inadequacy of current industrial approaches to identifying worst-case uncertain parameter combinations based on evaluating all combinations of minimum and maximum values of the parameters, (Fielding et al., 2002).

\subsection{Analysis $V$}

Analysis IV has already shown the significant effect of the uncertain parameters on the maximum AoA value when a specific fixed pilot input activity is considered. In this analysis both the pilot control inputs and $\Delta$ are searched simultaneously. The solution from this analysis therefore has two parts, one the pilot control inputs and the other the value of the uncertain parameters. The results are shown in Figure 4 and in Table 5. Figure 4 shows the pilot control input combination and the corresponding AoA time history. By the $6^{\text {th }}$ second the aircraft responses are outside the available aerodynamic database and hence the simulation stops. The worst-case combination of uncertainties is given in Table 5. The maximum AoA obtained is $69.3543^{\circ}$. Notice also that (a) the worst-case pilot control input is very different from the one computed in the previous analysis, and (b) the worst-case values of all the uncertain parameters are now located inside the searchspace hyperbox.

Finally, some explanation for the departure susceptibility of the aircraft for this particular sequence of pilot inputs can be found in Figure 5, which shows severe rate limiting of the rudder 


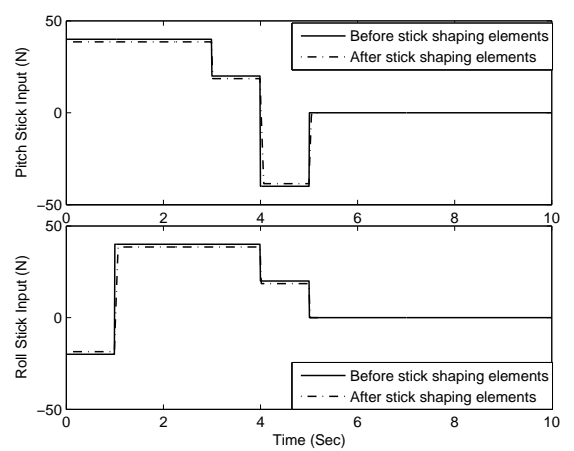

(a)

Fig. 4. Analysis V results

Table 5. Analysis V results

\begin{tabular}{ccccc|c}
\hline$\left[\begin{array}{ccccc}\Delta_{\text {mass }}^{*} & \Delta_{x c g}^{*} & \Delta_{C_{m_{\delta}}}^{*} & \Delta_{I_{y y}}^{*} & \Delta_{C_{m_{\alpha}}}^{*}\end{array}\right]$ & $\max \alpha(t)$ \\
\hline$\left[\begin{array}{lllll}0.0767 & 0.0739 & 0.0400 & 0.1856 & 0.0500\end{array}\right]$ & 69.36189 \\
\hline
\end{tabular}

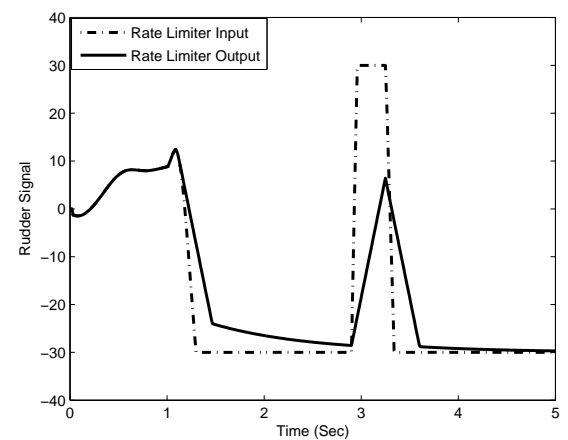

Fig. 5. Rudder path rate limiting in Analysis V

actuator. This suggests the need for further improvement of the current lateral/directional control law, and illustrates the contribution that this type of analysis can make to an iterative flight control law design cycle.

\section{CONCLUSIONS}

This paper has described an approach based on global optimisation and nonlinear simulation which may be used as part of the process of clearing a flight control law against departure susceptibility and/or violations of envelope protection limits. The flexibility of global optimisation methods is shown to allow for the simultaneous computation of worst-case pilot inputs and worstcase combinations of uncertain parameters in the nonlinear aircraft simulation model. The results show that only such a simultaneous consideration of worst-case pilot inputs and uncertain parameters is likely to reveal the true worst-case behaviour of the aircraft.

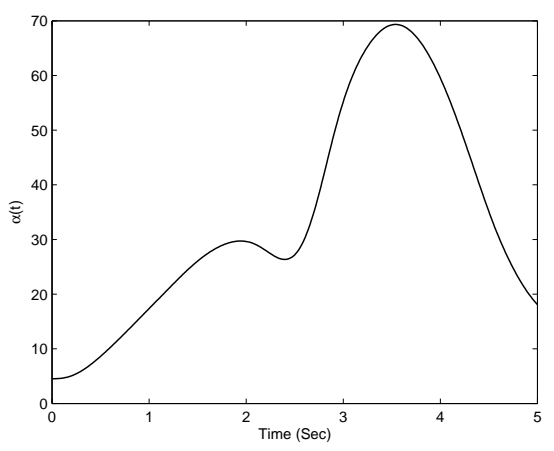

(b)

\section{ACKNOWLEDGEMENTS}

This work was carried out under EPSRC research grant GR/S61874/01. The authors are grateful to the members of GARTEUR FM-AG11 and Lars Forssell at FOI for many helpful discussions during the course of this research.

\section{REFERENCES}

Chetty, S., Deodhare G. and Misra B. B. (2002). Design, development and flight testing of control laws for the Indian Light Combat Aircraft. In: Proc. AIAA Atmospheric FM Conference. CP-3810, CA, USA.

Fielding, C., Varga A., Bennani S. and Selier M. (Eds.) (2002). Advanced Techniques for Clearance of Flight Control Laws. Springer.

Fleming, P. J. and R. C. Purshouse (2002). Evolutionary algorithms in control systems engineering: a survey. CEP. 10, 1223-1241.

Forssell, L. S. and Å Hyden (2003). Flight control system validation using global nonlinear optimisation algorithms. In: Proc. of ECC.

Forssell, L. S., Hovmark G., Hyden A. and Johansson F. (2001). The aero-data model in a research environment (ADMIRE) for flight control robustness evaluation. Technical Report GARTUER/TP-119-7.

Forssell, Lars (2004). Personal communication.

Goldberg, D. E. (1989). Genetic Algorithms in Search, Optimization and Machine Learning. Addison-Wesley.

Menon, P. P., Pashilkar A. A and Sudhakar K. (2003). Identification of departure susceptibility for design of carefree maneuverable control scheme. In: Proc. of the MSO-DMES Conference. Vol. 1. Paper No.77, Goa, India.

RyanIII, G. W. (1995). A genetic search technique for identification of aircraft departures. In: Proc. AIAA Atmospheric FM Conference. AIAA 95-3453, MD, USA. 